\title{
Ensemble experiments using a nested LETKF system to reproduce intense vortices associated with tornadoes of 6 May 2012 in Japan
}

\author{
Hiromu Seko ${ }^{1,2^{*}}$, Masaru Kunii ${ }^{1}$, Sho Yokota', Tadashi Tsuyuki ${ }^{4,1}$ and Takemasa Miyoshi ${ }^{3}$
}

\begin{abstract}
Experiments simulating intense vortices associated with tornadoes that occurred on 6 May 2012 on the Kanto Plain, Japan, were performed with a nested local ensemble transform Kalman filter (LETKF) system. Intense vortices were reproduced by downscale experiments with a 12-member ensemble in which the initial conditions were obtained from the nested LETKF system analyses. The downscale experiments successfully generated intense vortices in three regions similar to the observed vortices, whereas only one tornado was reproduced by a deterministic forecast. The intense vorticity of the strongest tornado, which was observed in the southernmost region, was successfully reproduced by 10 of the 12 ensemble members. An examination of the results of the ensemble downscale experiments showed that the duration of intense vorticities tended to be longer when the vertical shear of the horizontal wind was larger and the lower airflow was more humid. Overall, the study results show that ensemble forecasts have the following merits: (1) probabilistic forecasts of the outbreak of intense vortices associated with tornadoes are possible; (2) the miss rate of outbreaks should decrease; and (3) environmental factors favoring outbreaks can be obtained by comparing the multiple possible scenarios of the ensemble forecasts.
\end{abstract}

Keywords: Ensemble forecast, Tornadoes, LETKF

\section{Background}

Local heavy rainfalls and tornadoes cause severe damage due to flash floods, landslides, and strong winds. Accurate forecasts of these phenomena, for example, by numerical models, could reduce the damages they cause. However, numerical models are not perfect, and the initial conditions of numerical forecasts include errors. In particular, forecasts of convection cells generated in areas of weak convergence, which frequently occur in urban areas such as the Tokyo Metropolitan Area in summer, are affected by small errors in the initial conditions. This means that forecasts of the position and timing at which a thunderstorm will be generated from a weak convergence are

\footnotetext{
* Correspondence: hseko@mri-jma.go.jp

'Meteorological Research Institute, Japan Meteorological Agency, 1-1

Nagamine, Tsukuba, Ibaraki 305-0052, Japan

${ }^{2} J a p a n$ Agency for Marine-Earth Science and Technology, 3173-25,

Showa-machi, Kanazawa-ku, Yokohama, Kanagawa 236-0001, Japan

Full list of author information is available at the end of the article
}

sensitive to errors in the initial conditions. Because of the resulting uncertainty in forecasts of convection cell generation, ensemble forecast techniques must be employed to produce probabilistic forecasts. In addition to probabilistic forecasts, ensemble forecasts produce multiple possible scenarios. Thus, ensemble forecasts have the following merits: (1) analyzed fields of ensemble forecasts (i.e., the ensemble average of multiple scenarios) are statistically more accurate than those of deterministic forecasts and (2) multiple possible scenarios provide information regarding uncertainty (i.e., when there is large scatter among the scenarios, the uncertainty is larger).

The ensemble forecasts operationally performed by the Japan Meteorological Agency (JMA) with the highest horizontal resolution are typhoon forecasts and 1 week forecasts. However, these ensemble forecasts cannot represent convection cells of thunderstorms that cause local heavy rainfall and tornadoes because the horizontal grid interval $(40 \mathrm{~km})$ is too large to resolve the convection

\section{勿 Springer}


cells. Recently, several ensemble forecast experiments in which heavy rainfall or local heavy rainfall was reproduced have been performed with a local ensemble transform Kalman filter (LETKF; Hunt et al. 2007; Miyoshi and Aranami 2006). For instance, Kunii (2013) reproduced a heavy rainfall in northern Kyushu by assimilating JMA's operational observation data with a LETKF system with a grid interval of $5 \mathrm{~km}$. Probability distributions for a $3 \mathrm{~h}$ rainfall amount exceeding $50 \mathrm{~mm}$ were obtained by changing the initial time of the ensemble forecasts. Heavy rainfall of the northern Kyushu heavy rainfall event could be forecast with high probability even when the initial time of the ensemble forecasts was $12 \mathrm{~h}$ before the event.

One method of representing convection cells is to perform downscale forecasts with the model with a grid interval of a few kilometers and to obtain the initial conditions by spatial and temporal interpolation of the analyzed or predicted fields of a data assimilation system. For example, a local heavy rainfall, which caused a flash flood in the Toga River in Kobe that swept away six people, was reproduced by downscale experiments with JMA's non-hydrostatic model (JMANHM), the initial fields of which were obtained with a LETKF system (Seko et al. 2011). Though the linear band of intense rainfall responsible for the local heavy rainfall was represented as a weak rainfall region by the NHM-LETKF system with a grid interval of $20 \mathrm{~km}$, downscale simulations with a grid interval of $1.6 \mathrm{~km}$ were successful in reproducing the linear rainfall band. This result is one example of a local heavy rainfall being reproduced by modifying the environment around the rainfall system through the assimilation of observed data.

A local heavy rainfall that occurred on the Osaka Plain on 5 September 2008 was reproduced by a nested LETKF system (Seko et al. 2013). In this experiment, high-resolution data (Doppler radar and GPS data) were assimilated to reproduce the local heavy rainfall. The number of ensemble members that reproduced the local heavy rainfall was increased by the assimilation of highresolution data, compared with the number when the analyzed fields were obtained with assimilating only low-resolution conventional data. The results of these ensemble experiments show that the use of a LETKF system is a promising approach to the analysis and prediction of local heavy rainfall events.

In this study, we applied a LETKF system to the prediction of intense vortices associated with tornadoes. To date, no numerical experiments have been conducted using ensemble Kalman filters with a horizontal resolution of a few kilometers to reproduce intense vortices associated with tornadoes occurring in Japan, although a number of studies have used ensemble Kalman filters to reproduce or to investigate tornadoes that occurred in the USA. For instance, Snook et al. (2011) showed that it was possible to obtain probabilistic forecasts by using an ensemble Kalman filter analysis system that assimilated Doppler radar data from the Collaborative Adaptive Sensing of the Atmosphere and Weather Surveillance Radar-1988 systems. Yussouf et al. (2013) conducted simulations with a mesoscale and convective scale ensemble data assimilation system consisting of the Weather Research and Forecasting model and an ensemble adjustment Kalman filter that used a double-moment microphysics scheme, and they successfully reproduced a supercell storm. Clark et al. (2013) investigated the relation between the path lengths of tornadoes and simulated updraft helicity by using an ensemble Kalman filter system with a horizontal grid interval of $1.25 \mathrm{~km}$. Recently, Snook et al. (2015) demonstrated that use of an ensemble Kalman filter system could provide skillful analyses and ensemble-based probabilistic forecasts of tornadic mesoscale convective systems in the USA.

Tornadoes in Japan are different from those in the USA; Japanese tornadoes have an intensity equal to or less than F3 on the Fujita scale, and the low-level atmosphere in Japan is much more humid. Ensemble experiments to reproduce Japanese tornadoes are desirable for improving the forecast accuracy of tornadoes in Japan and for understanding the generation mechanisms of Japanese tornadoes.

We applied a nested LETKF system to a tornado event on 6 May 2012, during which three tornadoes were generated on the Kanto Plain. To express the vortices associated with these tornadoes, we performed downscaling experiments using the JMANHM model (Saito et al. 2006) with a horizontal grid interval of $350 \mathrm{~m}$ (NHM-350) and the analyzed fields produced by the LETKF system. We expected the multiple possible scenarios obtained from these ensemble forecast experiments to be useful for inferring environmental factors favoring the generation of intense vortices.

The rest of this paper is organized as follows: In the "Methods" section, the tornadoes of 6 May 2012 are first briefly described, and then, the LETKF system and downscale forecast experiments are explained. In the "Results and discussion" section, we present the results of the ensemble downscale experiments and discuss the factors that favor the generation of the intense vortices associated with tornadoes. In the "Conclusions" section, we present our conclusions.

\section{Methods}

\section{Observed features of the tornadoes of 6 May 2012}

On 6 May 2012, three tornadoes occurred on the northern Kanto Plain in Japan. The surface weather map for 09:00 Japan Standard Time (JST $=$ UTC +9 ) on 6 May (Fig. 1a) shows that there was a low-pressure system 
(a)

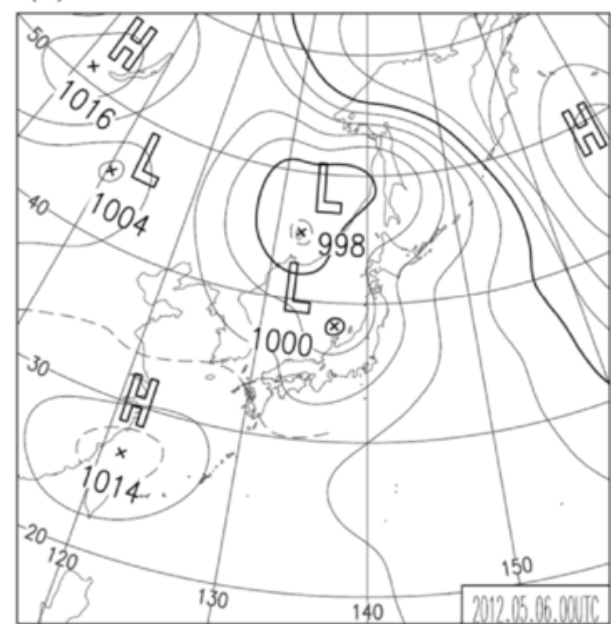

(b)

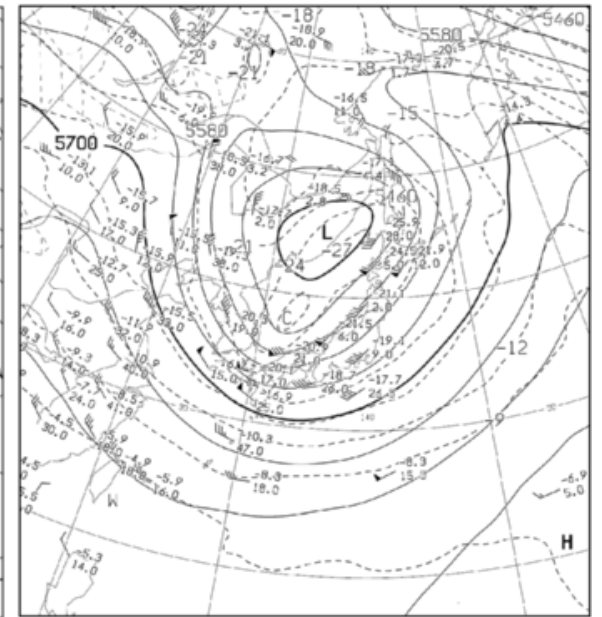

Fig. 1 a Surface weather map and $\mathbf{b}$ weather map at the height of $500 \mathrm{hPa}$ at 09:00 JST on 6 May 2012. b Solid and broken contours indicate height $(\mathrm{m})$ and temperature $\left({ }^{\circ} \mathrm{C}\right)$, respectively. Intervals of the solid and broken contours are $3^{\circ} \mathrm{C}$ and $60 \mathrm{~m}$, respectively

over eastern Russia and the Japan Sea. The presence of the low-pressure system over the Japan Sea caused the isobar contours over central Japan, including the Kanto Plain, to be relatively densely spaced. As a result, strong southerly winds were expected over the plain. At the same time, a low-pressure system existed at the $500 \mathrm{hPa}$ height that was centered in eastern Russia, at the same location as the center of the surface low-pressure system (Fig. 1b). Over the Japan Sea, there was a cold air mass with temperature lower than $-18{ }^{\circ} \mathrm{C}$, which was associated with this high-level low-pressure system. This cold air mass was moving southeast and approaching the Kanto Plain.

At 12:00 JST, a cloud region, visible in a satellite image, extended from southwest to northeast over central Japan (Fig. 2a). Because this cloud region was located along the eastern edge of the cold air mass at the height of $500 \mathrm{hPa}$, we deduced that it had been generated partly by the upper cold air mass as it approached the Kanto Plain, though surface temperature also contributed to the cloud generation. On the Kanto Plain, a brighter cloud area (A in Fig. 2a) indicated the presence of intense convection cells.

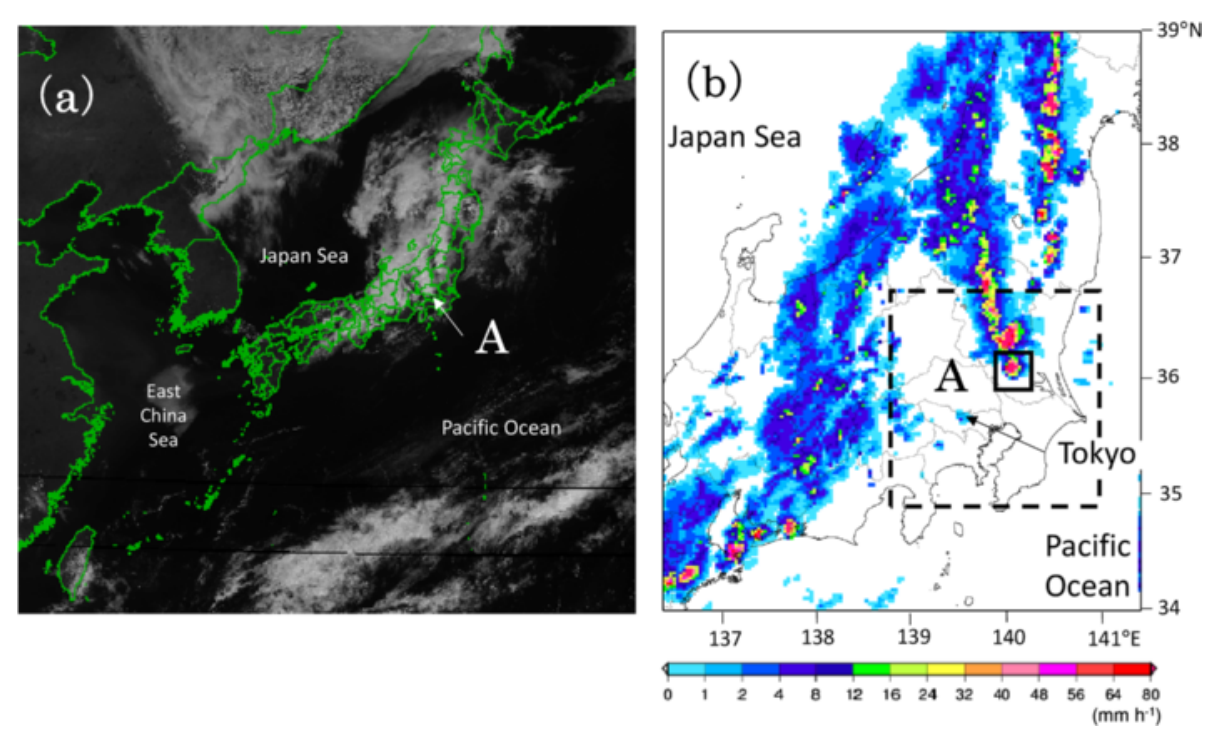

Fig. 2 aVisible image acquired at 12:00 JST on 6 May 2012 by a geostationary meteorological satellite of the Japan Meteorological Agency (JMA). b Rainfall intensity at 12:40 JST on 6 May 2012 observed by operational radars of JMA. The regions shown in Figs. 3 and 4 are indicated by the broken line and solid line rectangles, respectively 
At 12:40 JST, the operational radars of JMA showed that several rainfall regions (Fig. 2b), which corresponded to the cloud region over central Japan observed by satellite, extended northeastward from central Japan. In addition, another rainfall region (region A) that extended northward from the Kanto Plain corresponded to the bright cloud area seen over the northern Kanto Plain. Rainfall intensity in the southern part of region A in some places exceeded $80 \mathrm{~mm} \mathrm{~h}^{-1}$. These intense rainfall areas in region A were moving northeastward.

JMA determined the paths of the tornadoes that were generated on 6 May from the damage left in their wake (Japan Meteorological Agency, Meteorological Research Institute, Tokyo District Meteorological Observatory and Sendai District Meteorological Observatory 2012). Three tornadoes were generated at around 12:30 JST over the northern Kanto Plain. The intensity of the southernmost tornado was F3 on the Fujita scale, the highest intensity of tornadoes observed in Japan, and this tornado damaged about 800 houses, killed one person, and injured 37 people. The two northern tornadoes had intensities of F2 and F1.

Temperature and surface horizontal winds observed by JMA's Automated Meteorological Data Acquisition System (AMeDAS) showed that a warm southerly and a cold northerly airflow converged over the northern Kanto Plain on 6 May (Fig. 3). Rainfall regions in which rainfall intensity exceeded $56 \mathrm{~mm} \mathrm{~h}^{-1}$ were located in an area with a steep temperature gradient produced by the convergence that extended from southwest to northeast over the plain. This distribution indicates that these intense rainfall regions were produced by the convergence of the warm southerly flow and cold northerly flow. Just after the outbreak of the tornadoes (12:30 JST), two distinct regions of intense rainfall were observed (I and II in Fig. 3b). Wind speeds of the southerly flow east of these intense rainfall regions were markedly higher than wind speeds in the surrounding area. The tornado tracks (red lines in Fig. 3b) extended northeastward from near the southern edges of intense rainfall regions I and II.

Because the southernmost tornado passed near Narita city, the reflectivity and radial winds within the rainfall region in which the southernmost tornado was generated were observed by the Doppler radar at Narita International Airport (red dot in Fig. 3). A pairing of intense winds of opposite direction, indicating the mesocyclone of the southernmost tornado, was observed in a filament-like rainfall band (arrows in Fig. 4) at the southern end of the intense rainfall region. This configuration of a rainfall region with a tornado located near the filament-like rainfall band at its southern tip, resembling configurations that have been observed in the USA (figure 8.10 in Houze 1994), suggested that the rainfall system that generated the southernmost tornado had a classic supercell structure.

The Meteorological Research Institute (MRI) has analyzed the environmental conditions when these tornadoes were generated by using JMA's operational mesoscale analysis data (Meteorological Research Institute 2012). The water vapor distribution at 12:00 JST on 6 May showed that a moist airflow supplied the intense rainfall regions

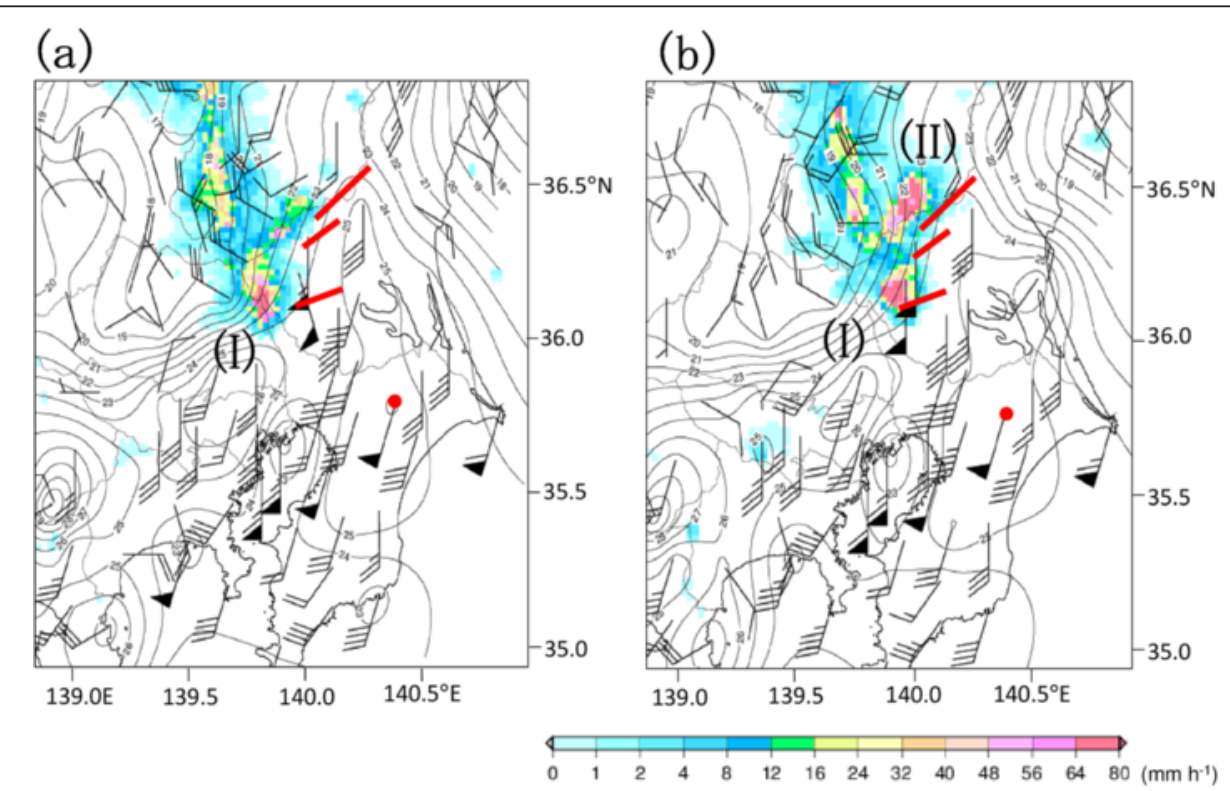

Fig. 3 Horizontal distributions of horizontal wind and temperature observed by AMeDAS at a 12:20 JST and b 12:30 JST on 6 May 2012 in the area enclosed by the broken line rectangle in Fig. 2. Colors indicate rainfall intensity observed by the operational radars of JMA. The red lines indicate the tornado tracks, as revealed by the damage they caused. The red dot in each panel indicates the position of the Narita radar 


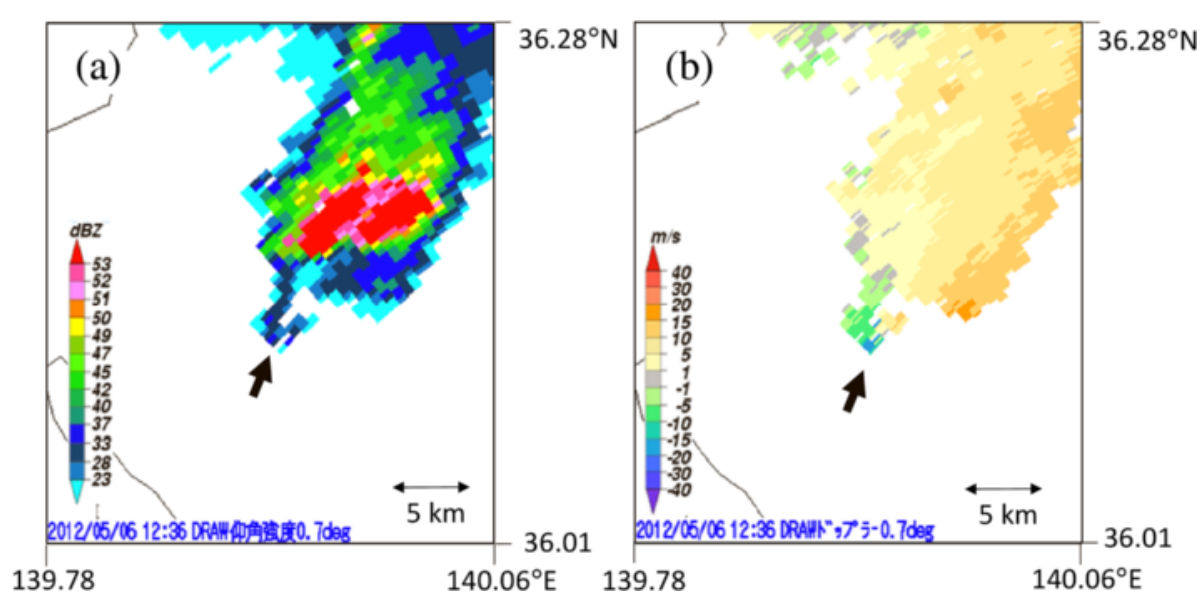

Fig. 4 Distributions of a rainfall intensity and $\mathbf{b}$ radial wind at 12:36 JST in the area within the solid line rectangle in Fig. 2, observed by the Narita airport radar (elevation angle $0.7^{\circ}$ ). The arrows indicate a filament-like rainfall band

where the tornadoes were generated. In particular, a moist airflow with a water vapor mixing ratio that exceeded $12 \mathrm{~g} \mathrm{~kg}^{-1}$ contributed to the generation of these tornadoes (Meteorological Research Institute 2012). To investigate the detailed structure of the convection cell that produced the southernmost tornado, MRI conducted a deterministic experiment using a model with a horizontal grid interval of $250 \mathrm{~m}$. Although this experiment reproduced only the southernmost tornado, it showed that the rainfall system that generated the tornadoes had a supercell structure. In the present study, we performed ensemble experiments to further investigate these three tornadoes generated on the northern Kanto Plain.

\section{Outline of the experiments performed with the nested LETKF system}

Downscale experiments were performed with NHM-350 to reproduce the intense vortices associated with the tornadoes. The initial conditions for NHM-350 were obtained from outputs of a nested LETKF system (Seko et al. 2013). The nested LETKF system (Fig. 5) was composed of two LETKF systems, each with 12 ensemble members; the grid interval of the outer LETKF was $15 \mathrm{~km}$ and that of the inner LETKF was about $1.875 \mathrm{~km}$. The outer LETKF was used to reproduce the large-scale convergence by assimilation of part of JMA's conventional data (horizontal wind, temperature, and relative humidity data from upper air soundings and aircraft and wind profiler observations and surface pressure data recorded by meteorological observatories). The outer LETKF domain consisted of $80 \times 80$ horizontal grids covering Japan, except for Hokkaido and Kyushu, and the domain of the inner LETKF, which was within the outer LETKF domain, consisted of $160 \times 160$ horizontal grids covering the Kanto Plain. The inner LETKF grid interval of $1.875 \mathrm{~km}$ was adopted so that the intense rainfall regions that generated the tornadoes could be represented. In both LETKFs, the number of vertical

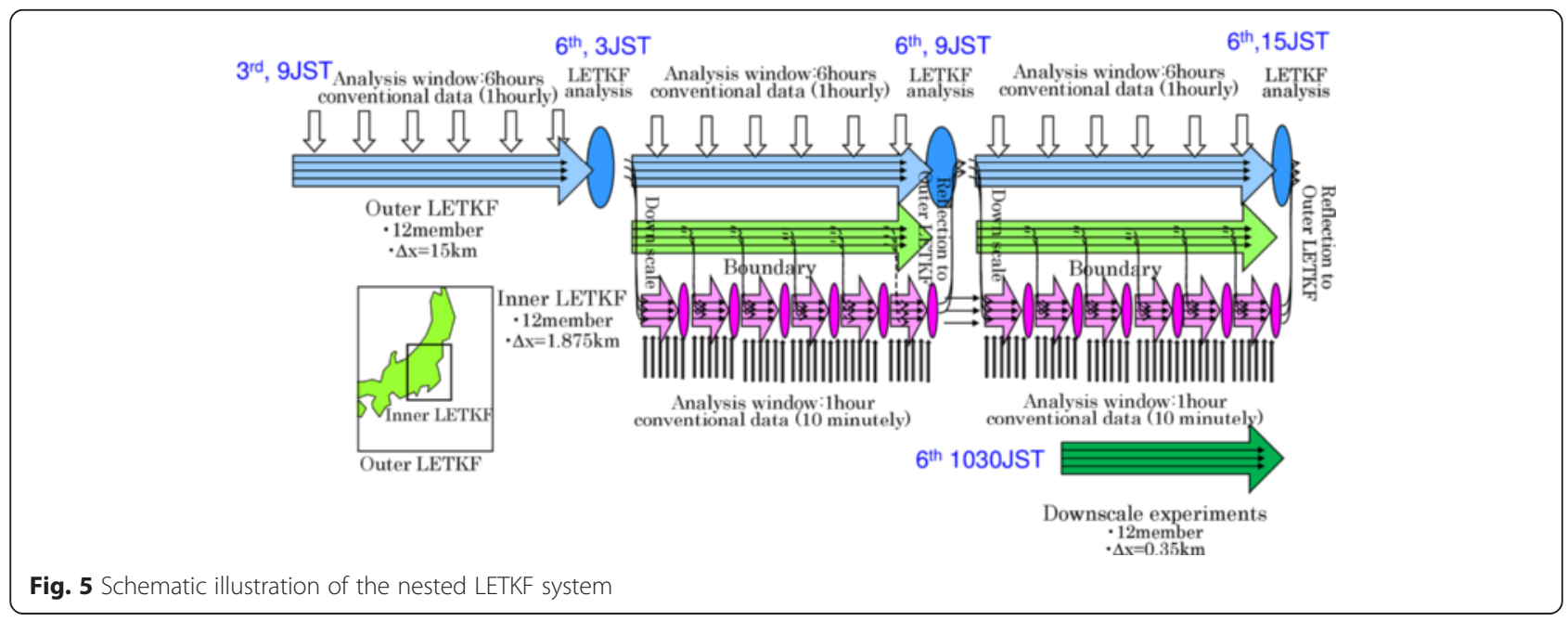


layers was 50, and the layer depth stretched from $20 \mathrm{~m}$ at the bottom to $890 \mathrm{~m}$ at the top of the domain. The altitude of the top was $22.6 \mathrm{~km}$. The assimilation window of the outer LETKF was $6 \mathrm{~h}$, and the data assimilation slot interval was $1 \mathrm{~h}$. To reduce the convergence time required to get the analyzed fields of the outer LETKF, initial seeds for the outer LETKF were obtained at several prior points in time from the initial time of the outer LETKF from analyzed fields of JMA's mesoscale model. Because analyzed fields were used to produce the boundary conditions of the outer LETKF, the results of this study are, strictly speaking, not forecasts but simulations of the tornadoes.

The assimilation window of the inner LETKF was $1 \mathrm{~h}$, and the slot interval was $10 \mathrm{~min}$. In this study, conventional data (described above) from JMA were assimilated in both LETKFs. Although higher resolution observation data (e.g., radial wind from Doppler radars and GPS precipitable water vapor) are expected to be better able to reproduce smaller scale distributions of the initial conditions than conventional data, we did not use such highresolution data in this study because our main purpose was to explore the merits of ensemble forecasts. Yokota et al. (2015) have shown that the assimilation of highresolution data (e.g., radial winds from Doppler radars and dense surface observation data of AMeDAS and the Environmental Sensor Network of NTT DOCOMO, Inc.) could improve the positions of the reproduced vortices of the tornadoes of 6 May 2012. The analyzed fields of the inner LETKF were reflected to those of the outer LETKF every $6 \mathrm{~h}$, at which time the analyzed fields of both the inner and outer LETKFs were available. The data assimilation cycles of the outer and inner LETKFs began at 09:00 JST on 3 September and 03:00 JST on 6 September, respectively.

NHM-350, which performed the downscale experiments to represent the intense vortices associated with the tornadoes, had $600 \times 600$ horizontal and 70 vertical grids, and its initial time was set to 10:30 JST on 6 September. The initial and boundary conditions for NHM-350 were produced from the output of the inner LETKF. NHM-350 used a double-moment microphysics parameterization scheme and the Deardorff (1980) planetary boundary scheme.

\section{Results and discussion}

Intense vortices produced by the inner LETKF and NHM-350 NHM-350 successfully reproduced the rainfall regions from 11:00 JST to 12:00 JST, which includes the period of the intense vortices associated with the tornadoes (Fig. 6). Because the rainfall regions were moving, the hourly rainfall region distribution extended northeastward over the northern Kanto Plain. An intense southerly flow from the south and a relatively weak flow under the relatively

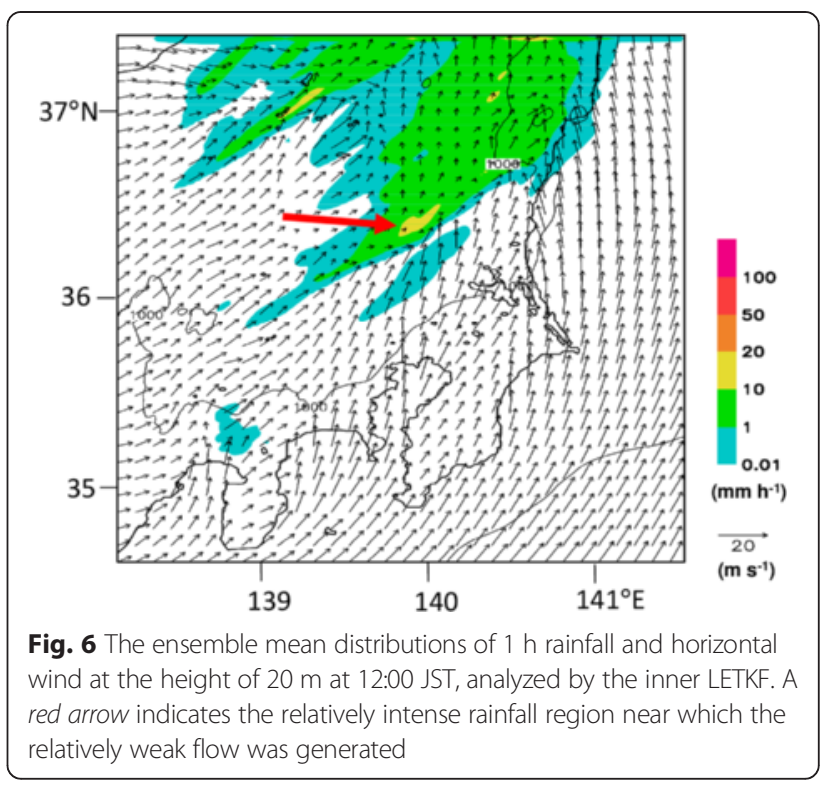

intense rainfall region (indicated by a red arrow in Fig. 6) were seen to converge along the southern and eastern edges of the rainfall regions. These distributions indicate that the rainfall regions were generated by this convergence. In general, this relationship between the rainfall regions and horizontal winds was the same as that observed, except that the northerly component of the airflow reproduced on the north side of the convergence line was much weaker than the observed airflow.

All 12 ensemble members of NHM-350 reproduced a few rainfall regions at 12:00 JST that extended to the north-northeast (Fig. 7a). As mentioned above, these rainfall regions were moving northeastward. The positions of the rainfall regions and the rainfall intensities varied among the ensemble members. Intense vortices, where vertical vorticity exceeded $0.1 \mathrm{~s}^{-1}$ at 12:00 JST (red dots in Fig. 7), were located near the southern tip of the rainfall regions generated by ensemble members $\# 000$, \#002, \#003, \#004, and \#008; this position corresponds to that of the southernmost tornado generated in intense rainfall region I (Fig. 3). In \#006, an intense vortex was generated near the southern tip of the northern rainfall region; this position corresponds to one of the northern tornadoes generated in the observed intense rainfall region II (Fig. 3).

Ensemble members \#004 and \#007 generated the strongest and weakest vorticities among the ensemble members, respectively, at the height of $20 \mathrm{~m}$. An intense vortex was generated near the southern tip of the intense convection by \#004 at 11:45 JST (Fig. 7b), 45 min earlier than the observed southernmost tornado was generated, but only weak vorticity was generated by \#007 (Fig. 7c). The position of the southernmost intense vortex relative to the rainfall region in \#004 was 


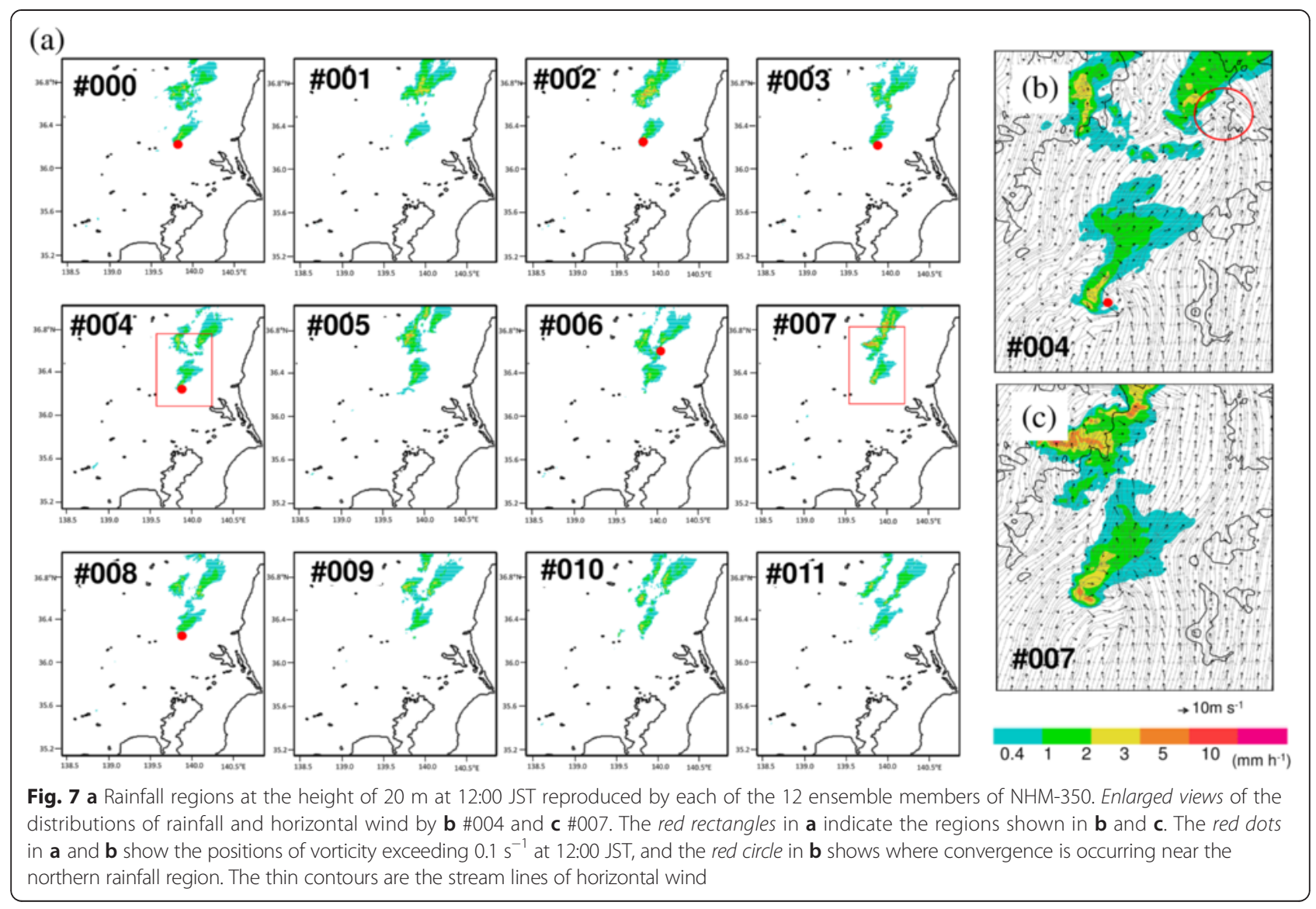

the same as that of the observed vortex relative to the observed rainfall region. This result indicates that \#004 reproduced the observed rainfall region that generated the tornado. An intense vorticity was not reproduced by \#007, even though \#007 produced more intense rainfall than \#004 did. The relationship between vorticity and rainfall intensity in \#007 suggests that rainfall intensity might not be a factor controlling the generation of intense vortices. The convergence reproduced by \#004 at the southern edge of the northern rainfall region (red circle in Fig. 7b) corresponds to the northern intense vortex that was reproduced by \#006 (Fig. 7a), though the vorticity generated by \#004 was weaker than $0.1 \mathrm{~s}^{-1}$. The stream lines, which show the small-scale distributions of horizontal wind, indicate that in \#004 (Fig. 7b) the airflow that converged at the position of the northern vortex passed near the southern rainfall region. If the southern rainfall region modified the airflows that were supplied to the northern rainfall region, it would prevent the development of the northern rainfall region.

We investigated the airflows that entered the northern rainfall region by performing a back trajectory analysis (Fig. 8). The end points of the tracers were positioned around the southern tip of the northern rainfall region at 12:00 JST and traced backward in time for $90 \mathrm{~min}$.
The tracers were assumed to move with the speed of the horizontal wind at the height of $0.8 \mathrm{~km}$, so that the paths of the air inflows before their arrival at the northern rainfall region could be used to check the influence of the southern rainfall region on the northern vortices.

We chose ensemble members \#002 and \#006 for the trajectory analysis because intense vorticity was generated near the northern rainfall region by $\# 006$ but not by \#002 (Fig. 7).

Both members showed most airflows approaching the southern tip of the northern rainfall region from the south. In \#002, the airflows passed the southern rainfall region at 11:30 JST, and their potential temperature decreased before their arrival at the northern rainfall region. In \#006, in contrast, some airflows passed far to the east of the southern rainfall region, and their temperature did not decrease. After the passage of these airflows from the east of the southern rainfall region, their direction of movement was changed by the presence of low pressure near the rainfall region, causing them to approach the southern tip of the northern rainfall region. That is, the southern rainfall region in \#006 is deduced to have a smaller effect on the airflows that were supplied to the northern rainfall region. This difference of the paths, as a result, might be one reason why \#006 was 


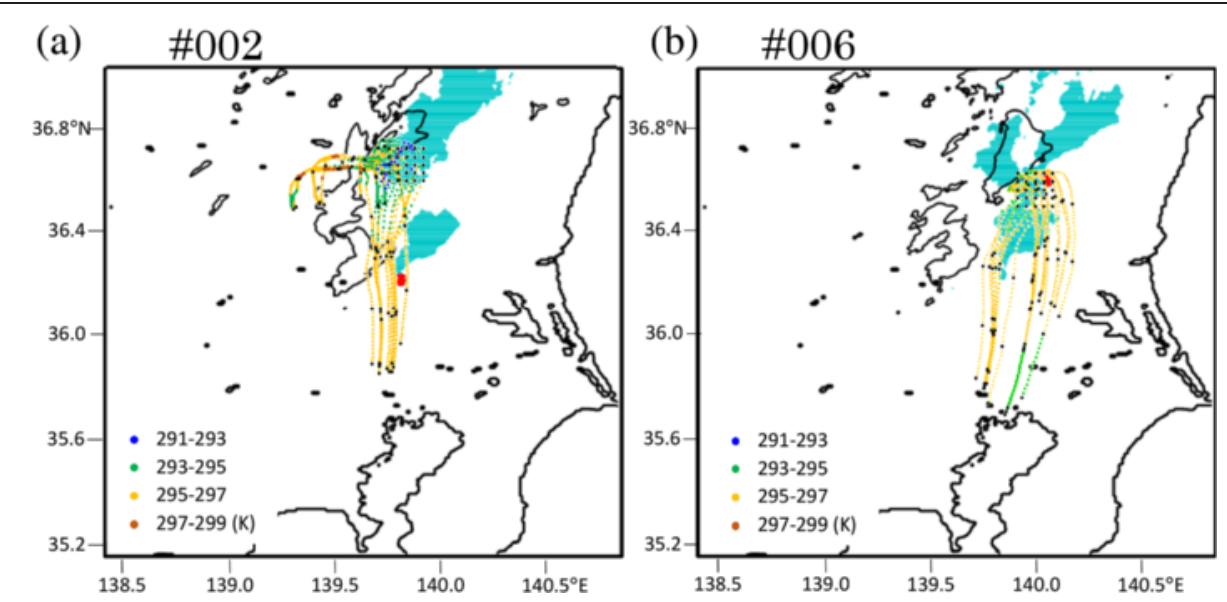

Fig. 8 Rainfall regions at the height of $20 \mathrm{~m}$ at 11:30 JST (contours) and 12:00 JST (blue regions) reproduced by two NHM-350 ensemble members: $\mathbf{a} \# 002$ and $\mathbf{b} \# 006$. The red dots in each panel show where vorticity exceeded $0.1 \mathrm{~s}^{-1}$ at $12: 00$ JST. The small dots show the trajectories traced backward in time from positions around the southern edge of the northern rainfall region. The colors of the dots indicate the potential temperature at the tracer positions. The black dots on the tracer paths show the tracer positions, from south to north, at 10:30, 11:00, 11:30, and 12:00 JST

able to generate intense vorticity near the northern rainfall region. This relationship between the southern rainfall and the intense vortex near the northern rainfall region was obtained with the NHM-350 system. To confirm the existence of a corresponding observed relationship, observation data of airflows within the lower atmosphere over the Kanto Plain would be needed.

The intense vortices, with vertical vorticity larger than $0.1 \mathrm{~s}^{-1}$, reproduced by the 12 ensemble members from 11:00 JST to 13:00 JST in the downscale experiments (colored dots in Fig. 9) occurred in the same three regions as the observed tornadoes (Fig. 3), though the precise positions of the observed tornadoes were not reproduced. The southernmost intense vorticity, which was also reproduced by the deterministic forecast mentioned above, was generated by most of the ensemble members. In fact, an intense vortex associated with the southernmost tornado was reproduced by 10 of the 12 ensemble members (thus, the occurrence probability of an intense vortex was $83 \%$ ). The ensemble experiment also reproduced the two northern intense vortices, though their generation probability was low. This result indicates that probabilistic forecasts of intense vortices associated with tornadoes are possible and that ensemble forecasts can decrease the number of tornado outbreaks that are missed. Among the ensemble members, the paths of the southernmost intense vortex were clustered, whereas those of the northern vortices were scattered. This result indicates that the uncertainty of the paths was larger in the case of the northern vortices than it was in the case of the southernmost vortex. The ability to obtain information on uncertainty is one of the merits of ensemble forecasts mentioned in the "Background" section.
Next, we compared the outputs of NHM-350 around the mature vortices among the ensemble members to investigate the environmental factors favoring the generation of the intense vortices. We focused on the relationship between the durations of intense vortices and the values of certain environmental factors, because a tornado with longer duration causes damage over a wider area (Fig. 10). Here, for each member, duration was defined as the period during which vorticities exceeded $0.1 \mathrm{~s}^{-1}$, and the environmental values (water vapor at $0.8 \mathrm{~km}$ height, vertical wind shear between the heights

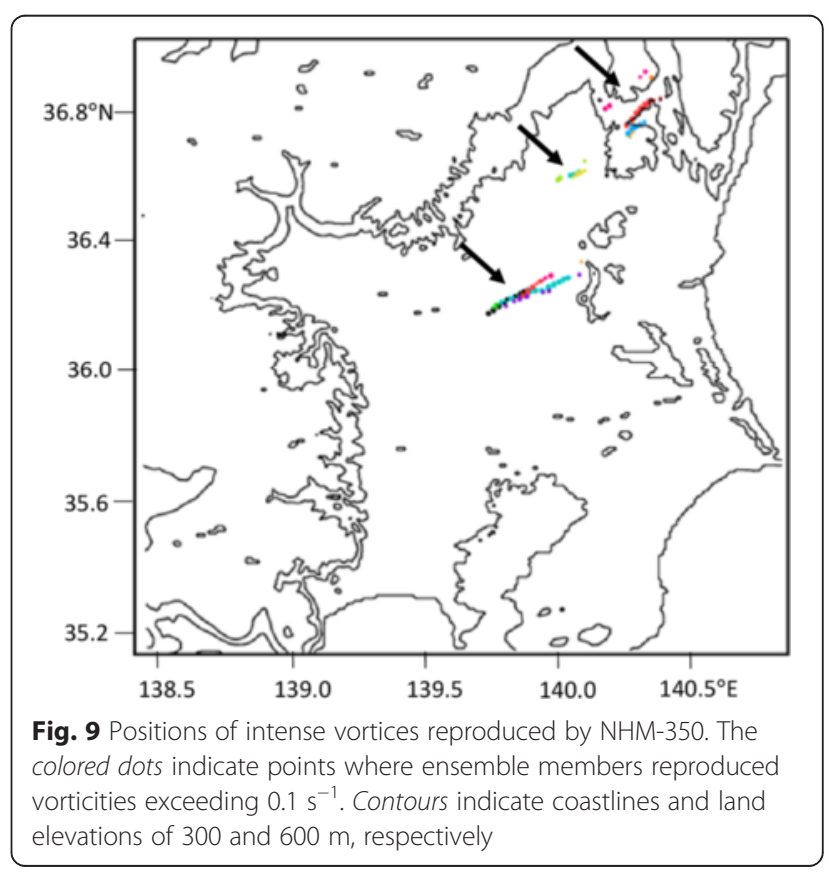




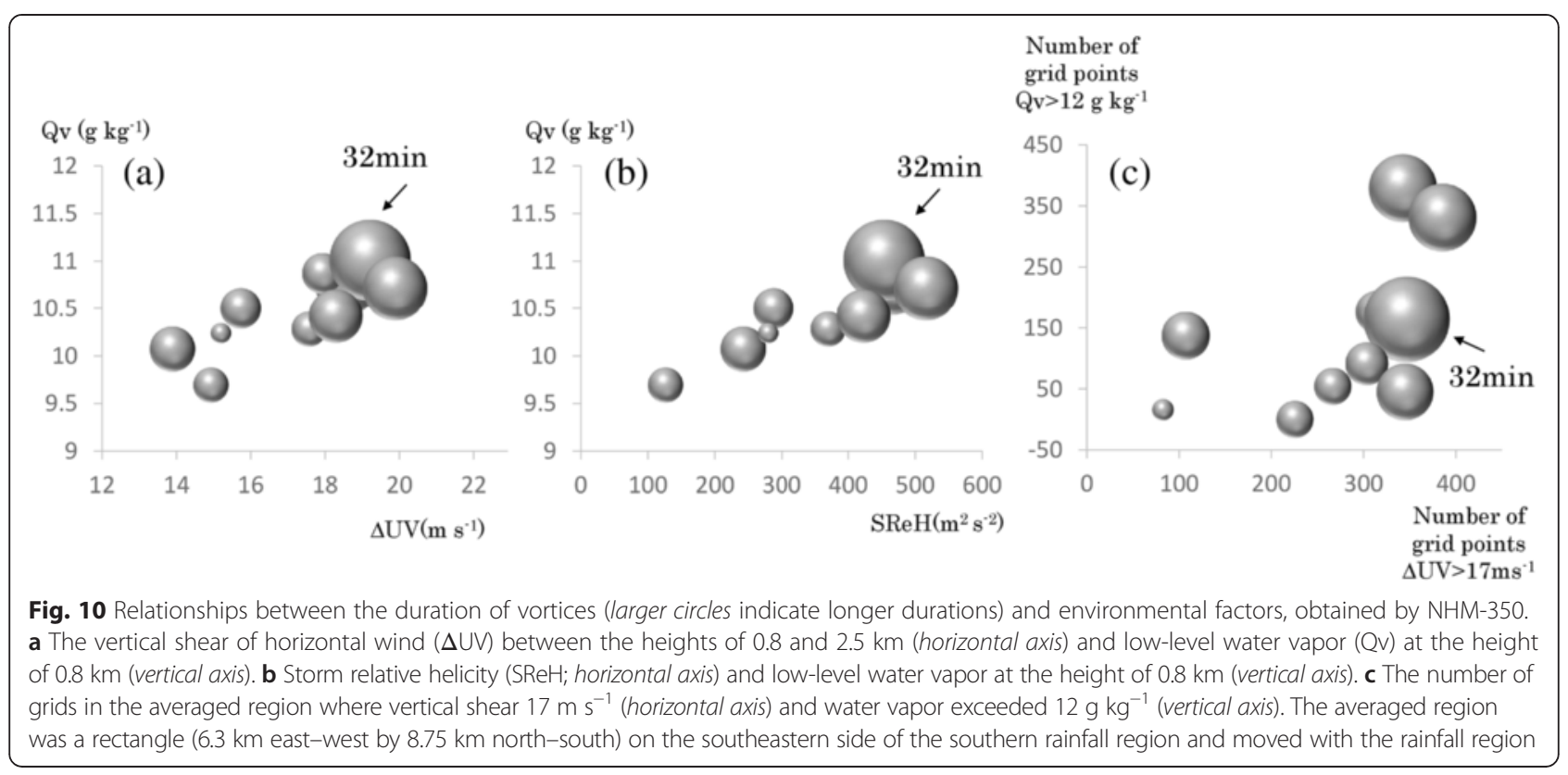

of 0.8 and $2.5 \mathrm{~km}$, and storm relative helicity (SReH)) are the spatial averages within a rectangle $(6.3 \mathrm{~km}$ eastwest $\times 8.75 \mathrm{~km}$ north-south) on the southeastern side of the southern rainfall region at the time the vorticities reached $0.1 \mathrm{~s}^{-1}$. This rectangle moved with the moving speed of the southern rainfall region. SReH is defined by the following equation:

$$
\mathrm{SReH}=\int_{0}^{3 \mathrm{~km}}\left[\frac{\partial \boldsymbol{V}(z)}{\partial z} \times(\boldsymbol{V}(z)-\boldsymbol{C})\right] \cdot \boldsymbol{k} \mathrm{dz}
$$

where $V(z)$ is the horizontal wind vector at height $z$, $\boldsymbol{k}$ is the vertical unit vector, and $C$ is the moving vector of the storm. To estimate $\mathrm{SReH}$, the moving speed $C$ of the southern rainfall region is needed. In this study, the moving speeds of the reproduced southern rainfall regions were used. A scatter diagram of the durations of intense vortices and the values of certain environmental factors shows that the duration became longer as the low-level layer became more humid and the vertical wind shear became larger (Fig. 10a). The duration also became longer when the $\mathrm{SReH}$ in the averaged region was larger.

In addition, comparison of duration with the number of grids in which water vapor and vertical wind shear in the averaged area exceeded $12 \mathrm{~g} \mathrm{~kg}^{-1}$ and $17 \mathrm{~m} \mathrm{~s}^{-1}$, respectively (Fig. 10c), showed that the durations of the intense vortices became longer as the number of grids exceeding these thresholds became larger. This result was expected because the number of grids in which values exceeded these thresholds was correlated positively with the averaged values shown in Fig. 10a. A larger number of grids with values exceeding these thresholds indicated that the favorable region for intense vortex generation within the averaged area, that is, within the rectangle on the inflow side of the southern rainfall region, was larger. Therefore, these results indicate that ensemble members reproducing large areas with a favorable environment reproduce vortices with a long duration. Thus, favorable environmental factors can be extracted by comparing the outputs of the ensemble members.

Environmental factors affecting intense vortex generation The temporal variations of intense vortices and the horizontal distributions of environmental factors (e.g., lowlevel water vapor and vertical shear of horizontal winds) are examined in this section to show how environmental factors affect intense vortex generation. Figure 11 shows the temporal variations of maximum vorticity obtained by NHM-350 within the whole domain of NHM-350 from forecast time (FT) $=0 \mathrm{~min}$ to $180 \mathrm{~min}$. Because the vorticities of ensemble members \#004 and \#007 were the strongest and weakest, respectively, we expected them to provide information about the key factors that affect the outbreak of intense vortices. To show the impacts of environmental factors on the intensity of vertical vorticities more clearly, we performed a series of experiments in which the initial conditions were produced by combining the initial conditions of \#004 and \#007 with different weights as follows:

$$
I_{\text {combined }}=w \times I_{004}+(1-w) \times I_{007}
$$

where $w$ is the weight of ensemble member \#004. 


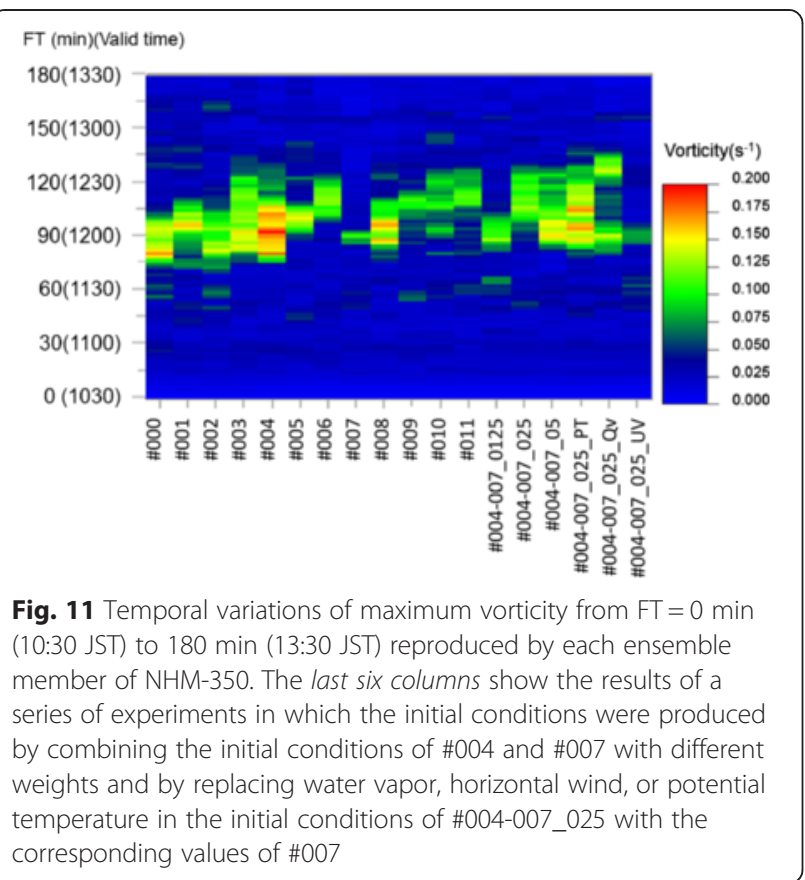

As $w$ became smaller, the maximum vorticity generated during FT = 0 min to $180 \mathrm{~min}$ became weaker (Fig. 11). This result is expected because the impact of \#004 is reduced when $w$ is smaller; the timing of the maximum vorticity, however, did not change linearly from that of $\# 007$ as $w$ decreased. When the weight was changed from 0.25 (\#004-007_025) to 0.125 (\#004-007_0125), the vorticity decreased greatly. This large change of vertical vorticity suggests that the \#004-007_025 experiment was particularly sensitive to environmental factors; therefore, we expected this experiment to show the impact of environmental factors more clearly than the others. Thus, we investigated the impact of each environmental factor by replacing water vapor, horizontal wind, or potential temperature in the initial conditions of \#004-007_025 with those of \#007.

When the horizontal wind or water vapor was replaced with those of \#007 (\#004-007_025_UV, \#004007_025_QV), the vorticities became weaker. In contrast, when the potential temperature was replaced with that of \#007 (\#004-007_025_PT), vorticity became more intense. These results indicate that the horizontal wind and water vapor in the initial conditions of \#004 were favorable for the generation of an intense vortex but that the temperature distribution of \#004 suppressed the generation of an intense vortex.

We therefore examined the horizontal distributions of the vertical shear of horizontal winds and of the lowlevel water vapor at 11:40 JST (5 min before the outbreak of the reproduced southernmost intense vortex in $\# 004$ ) reproduced by $\# 004$ and $\# 007$ to see where the differences existed between them (Fig. 12). On the southeastern side of the rainfall regions, that is, on the inflow side of the southernmost intense vortex, both $\# 004$ and \#007 simulated an intense vertical shear region where wind shear between the heights of 0.8 and $2.5 \mathrm{~km}$ was larger than $12 \mathrm{~m} \mathrm{~s}^{-1}$ (circles in Fig. 12a, b). As this region of intense vertical shear moved northward and the rainfall regions approached it from the west, the intensity of the vertical shear became larger. This region in which vertical shear exceeded $12 \mathrm{~m} \mathrm{~s}^{-1}$, however, was larger in \#004 than in \#007 (Fig. 12a, b). This result suggests that the intensity of vertical shear affects the outbreak of intense vortices, with the likelihood of an outbreak increasing with the intensity of vertical shear. The distributions of low-level water vapor show that a humid region also existed on the eastern side of the rainfall regions in both members \#004 and \#007 and that more water vapor was supplied to the rainfall regions in \#004 than in \#007 (Fig. 12c, d). This difference in lowlevel water vapor between \#004 and \#007 might be one reason why intense vorticity was not generated in \#007.

The temporal variations of water vapor and vertical shear of horizontal wind on the southeastern side of the southernmost intense vortex in \#004 are compared with those of vorticity in Fig. 13. The estimation region of the temporal variations was the same as that used in producing Fig. 10. The water vapor supplied to the rainfall region gradually increased up until the outbreak of the southernmost intense vortex (outbreak time in \#004, 11:45 JST), though there was a relatively small water vapor period from 11:30 JST to 11:40 JST. The vorticity became rapidly larger just after an abrupt increase in the vertical shear of the horizontal winds. These temporal variations in water vapor and vertical shear show that, in the case of this event, the intense vertical shear determined the timing of the outbreak of intense vorticity.

\section{Conclusions}

The intense vortices associated with tornadoes occurring on 6 May 2012 were reproduced by a nested LETKF system that simulated the environments and convection cells simultaneously. The results of this study demonstrated three merits of ensemble forecasts based on the outputs of the nested LETKF system. The occurrence probability of an intense vortex with a vertical vorticity exceeding $0.1 \mathrm{~s}^{-1}$ during this tornado event was $83 \%$. This result shows the first merit: probabilistic forecasts of the outbreak of intense vortices associated with tornadoes are possible. In addition, the multiple possible scenarios revealed two other merits of ensemble forecasts. Although a deterministic forecast had reproduced only the southernmost of the three tornadoes, several ensemble members reproduced the intense vortices associated with the two northern tornadoes. Thus, the second merit is that ensemble forecasts can be expected to decrease 

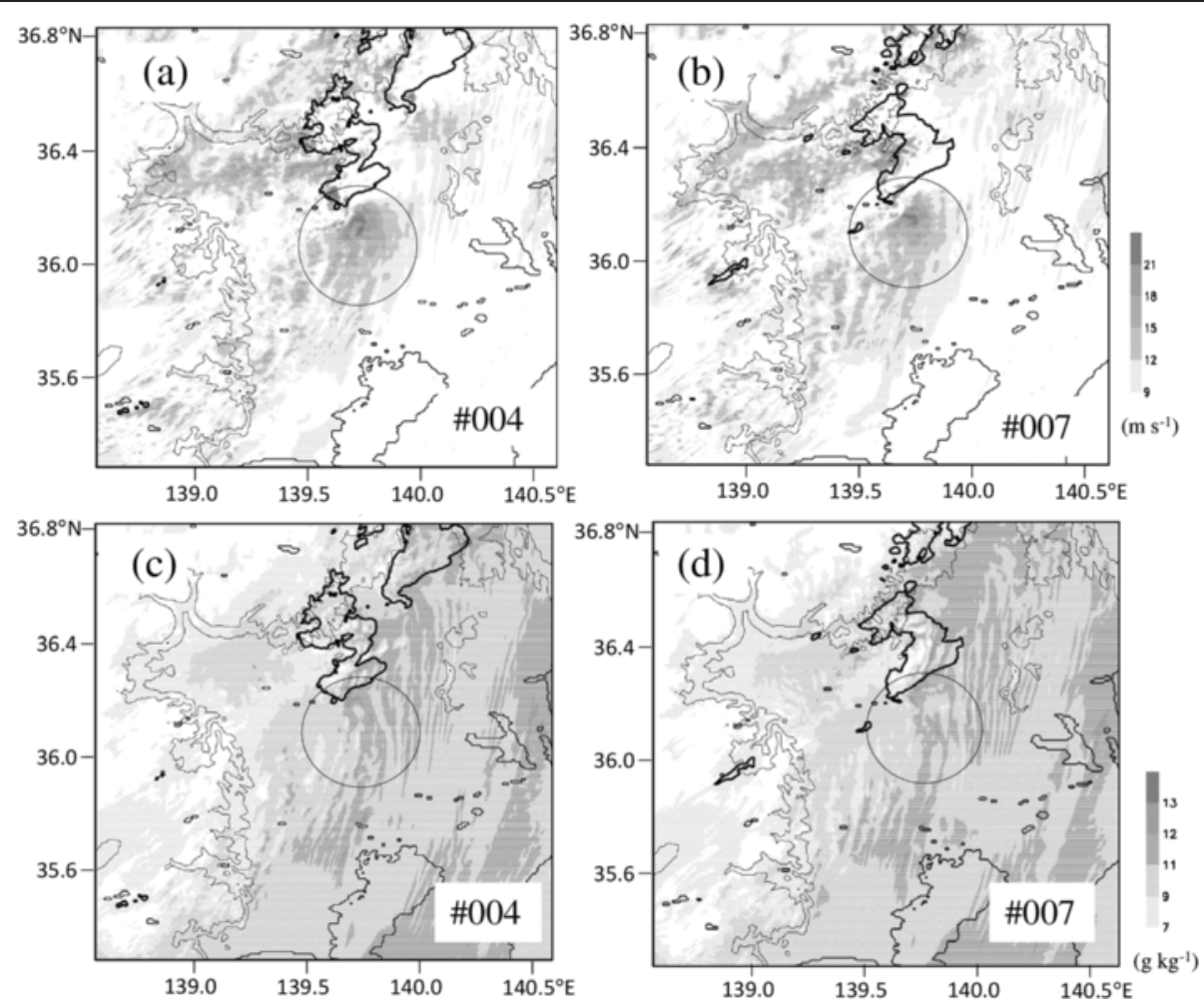

Fig. 12 Vertical shear of horizontal wind between the heights of 2.5 and $0.8 \mathrm{~km}$ at FT = $70 \mathrm{~min}$ (11:40 JST) in $\mathbf{a}$ \#004 and $\mathbf{b}$ \#007 and the horizontal distribution of the water vapor mixing ratio at the height of $0.8 \mathrm{~km}$ at FT $=70 \mathrm{~min}(11: 40 \mathrm{JST})$ in $\mathbf{c} \# 004$ and $\mathbf{d}$ \#007. The thick black contours indicate a rain mixing ratio of $1.0 \mathrm{~g} \mathrm{~kg}^{-1}$. The circles indicate the area in which airflows were supplied to the rainfall regions

the miss rate of outbreaks because they can reproduce low-probability phenomena. Comparison of the multiple possible scenarios showed that the duration of the southernmost tornado was related to the low-level water vapor and the vertical wind shear between heights of 0.8 and $2.5 \mathrm{~km}$. Thus, the third merit is that the multiple possible scenarios of the ensemble forecast make it possible to determine the environmental factors favorable for the formation of severe weather phenomena such as intense vortices associated with tornadoes.

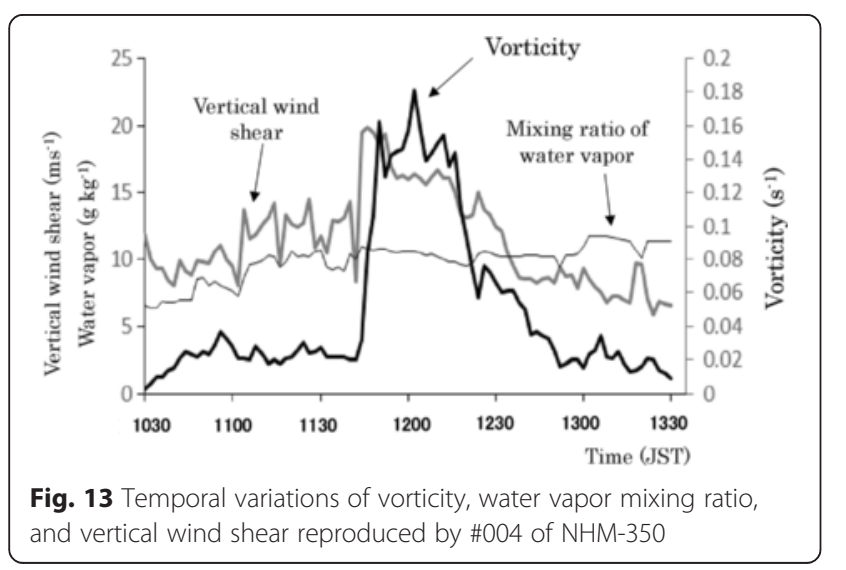

Although we demonstrated the merits of ensemble forecasts in this paper, there are several areas where improvements are needed. First, the initial seeds used might influence the probability of outbreaks of intense vortices. In this study, the initial seeds of the outer LETKF were provided by JMA's mesoscale model analysis fields from before the occurrence of the observed tornadoes, not by adding random perturbations. In this experiment, differences among the initial seeds might have been too small to cause dispersion of intense vortices to be large.

The second area is the number of ensemble members. The merits of the ensemble forecasts of convective scale phenomena, such as the possible distribution of intense vortices, can be demonstrated with as few as 12 ensemble members. For quantitative discussion, however, more ensemble members would be preferable. In this study, we investigated the relationships between environmental parameters and intense vortices in each ensemble member, but we did not discuss the ensemble mean distributions in depth. As shown in Fig. 3, a region of intense southerly winds existed east of the rainfall regions. Because of the small ensemble size, the ensemble mean distribution, if it was produced by simple averaging of the outputs of the ensemble members, would show several separate regions of intense southerly winds; 
as a result, the relationships between the southerly wind and the rainfall regions would be obscured. Therefore, the relationships in this study were extracted from the output of each ensemble member. It would also be possible to extract these relationships from composite distributions relative to the positions and timings of the occurrence of intense vortices; this approach was discussed by Yokota et al. (2014).

The third area is the assimilation data of the inner LETKF. High-resolution and high-frequency data are needed to reproduce convection cells in rainfall regions. Because such data were not used in this study, the intense vortices were not reproduced at the positions where they were actually observed. For more realistic reproduction of rainfall regions and tornadoes, highresolution and high-frequency data should be used.

\section{Abbreviations}

AMeDAS: Automated Meteorological Data Acquisition System; JMA: Japan Meteorological Agency; JMANHM: Japan Meteorological Agency non-hydrostatic model; JST: Japan Standard Time; LETKF: Local Ensemble Transform Kalman Filter; MRI: Meteorological Research Institute; NHM-350: Non-Hydrostatic Model with a grid interval of $350 \mathrm{~m}$.

\section{Competing interests}

The authors declare that they have no competing interests.

\section{Authors' contributions}

HS, T, MK, and TM developed the nested LETKF system. HS performed the analyses obtained from the nested LETKF and downscale experiments. HS and SY developed the interpretations. All authors read and approved the final manuscript.

\section{Acknowledgements}

The authors express their gratitude to Drs. Kazuo Saito, Teruyuki Kato, and Wataru Mashiko of MRI, to Mr. Hiroshi Yamauchi of JMA, and to two anonymous reviewers for valuable comments. Mesoscale analysis data and conventional observation data from JMA were used to produce the initial seeds and boundary conditions of the outer LETKF and in the data assimilation. "Kasaneru 3D" and "Multi-screen monitoring tool" were used to produce Figs. 2, 4, and 5. The authors extend their gratitude to the Tokyo District Meteorological Observatory and the Observation Department and Numerical Prediction Division of JMA. This study was supported in part by "Strategic Programs for Innovative Research (SPIRE), Field 3 (ID: hp120282, hp130012, hp140220)" and "Tokyo Metropolitan Area Convection Study for Extreme Weather Resilient Cities (TOMACS)." Science section to which this research article belongs: 2) Atmospheric and hydrospheric sciences

\begin{abstract}
Author details
${ }^{1}$ Meteorological Research Institute, Japan Meteorological Agency, 1-1 Nagamine, Tsukuba, Ibaraki 305-0052, Japan. ${ }^{2} J a p a n$ Agency for Marine-Earth Science and Technology, 3173-25, Showa-machi, Kanazawa-ku, Yokohama, Kanagawa 236-0001, Japan. ${ }^{3}$ RIKEN Advanced Institute for Computational Science, 7-1-26, Minatojima-minami-machi, Chuo-ku, Kobe, Hyogo 650-0047, Japan. ${ }^{4}$ Meteorological College, 7-4-81 Asahicho, Kashiwa, Chiba 277-0852, Japan.
\end{abstract}

Received: 22 April 2015 Accepted: 5 November 2015

Published online: 26 November 2015

\section{References}

Clark AJ, Gao J, Marth PT, Smith T, Kain JS, Correia Jr J, et al. Tornado pathlength forecasts from 2010 to 2011 using ensemble updraft helicity. Monthly Weather Review. 2013;28:387-407.
Deardorff JW. Stratocumulus-capped mixed layers derived from a three-dimensional model. Bound-Layer Meteor. 1980;18:495-527.

Houze Jr RA. Cloud dynamics. San Diego: Academic Press; 1994.

Hunt BR, Kostelich EJ, Szunyogh I. Efficient data assimilation for spatiotemporal chaos: a local ensemble transform Kalman filter. Physica D: Nonlinear Phenomena. 2007. 230:112-126.

Japan Meteorological Agency, Meteorological Research Institute, Tokyo District Meteorological Observatory and Sendai District Meteorological Observatory. Tornadoes generated on $6^{\text {th }}$ May 2012. 2012. http://www.jma.go.jp/jma/menu/ tatsumaki-portal/tyousa-houkoku.pdf, Accessed 21 July 2014 (in Japanese).

Kunii M. Mesoscale data assimilation for a local severe rainfall event with the NHM-LETKF system. Weather and Forecasting. 2013;29:1093-105.

Meteorological Research Institute. Tornadoes generated near Tsukuba, Ibaraki on 6th May 2012. 2012. http://www.jma.go.jp/jma/press/1205/11c/120511tsukuba_ tornado.pdf, Accessed 21 July 2014 (in Japanese).

Miyoshi T, Aranami K. Applying a four-dimensional local ensemble transform Kalman filter (4D-LETKF) to the JMA nonhydrostatic model (NHM). SOLA. 2006;2:128-31.

Saito K, Fujita T, Yamada Y, Ishida J, Kumagai Y, Aranami K, et al. The operational JMA nonhydrostatic mesoscale model. Monthly Weather Review. 2006;134: 1266-98.

Seko H, Miyoshi T, Shoji Y, Saito K. Data assimilation experiments of precipitable water vapour using the LETKF system: intense rainfall event over Japan 28 July 2008. Tellus A. 2011;63:402-14.

Seko H, Tsuyuki T, Saito K, Miyoshi T. Development of a two-way nested-LETKF system for cloud-resolving model. In: Park SK, Xu L, editors. Data assimilation for atmospheric, oceanic and hydrological applications, vol. 2. Springer: Heidelberg; 2013. p. 489-507.

Snook N, Xue M, Jung Y. Analysis of a tornadic mesoscale convective vortex based on ensemble Kalman filter assimilation of CASA X-band and WSR-88D Radar data. Monthly Weather Review. 2011;139:3446-68.

Snook N, Xue M, Jung Y. Multiscale EnKF assimilation of radar and conventional observations and ensemble forecasting for a tornadic mesoscale convective system. Monthly Weather Review. 2015;143:1035-57.

Yokota S, Kunii M, Seko H. Doppler radar radial wind assimilation for the tornado outbreak on May 6, 2012. CAS/JSC WGNE Research Activities in Atmospheric and Oceanic Modelling. 2014;1:29-1.30.

Yokota S, Kunii M, Seko H, Yamauchi H. Assimilation of rainwater estimated by the polarimetric radar for tornado outbreaks on 6 May 2012. CAS/JSC WGNE Research Activities in Atmospheric and Oceanic Modelling. 2015;1:27-1.28.

Yussouf N, Mansell ER, Wicker LJ, Wheatley DM, Stensrud DJ. The ensemble Kalman filter analyses and forecasts of the 8 May 2003 Oklahoma City tornadic supercell storm using single- and double-moment microphysics schemes. Monthly Weather Review. 2013;141:3388-412.

\section{Submit your manuscript to a SpringerOpen ${ }^{\circ}$ journal and benefit from:}

- Convenient online submission

- Rigorous peer review

- Immediate publication on acceptance

- Open access: articles freely available online

- High visibility within the field

- Retaining the copyright to your article

Submit your next manuscript at $>$ springeropen.com 